\title{
Meeting Increased Demand for THA and Follow-Up: An Actuarial Method to Determine Optimal Follow-Up Schedules
}

\author{
John B. Meding1,2, Merrill A. Ritter ${ }^{1,2}$, Kenneth E. Davis ${ }^{1,2}$, Alex Farris ${ }^{1,2}$, \\ Tatsuya Sueyoshi1,2 \\ ${ }^{1}$ Indiana Research Foundation (JRSI), Center for Hip and Knee Surgery, Mooresville, IN, USA \\ ${ }^{2}$ Midwest Center for Joint Replacement, Indianapolis, IN, USA \\ Email: jrsiresearch1@gmail.com
}

Received 15 June 2015; accepted 14 August 2015; published 17 August 2015

Copyright (C) 2015 by authors and Scientific Research Publishing Inc.

This work is licensed under the Creative Commons Attribution International License (CC BY). http://creativecommons.org/licenses/by/4.0/

c) (i) Open Access

\begin{abstract}
If a surgeon performs 200 procedures per year, he/she will have to see 800 patients for follow-up by year 5 and 1300 patients per year by year 10. Normal time constraints make this implausible. When do total hip arthroplasty (THA) patients have the greatest need for follow-up? We reviewed 8331 primary THAs to determine the greatest risk of failure across time. Patients failed with the greatest ratio at 1 year or earlier, followed by 10 and 12 years postoperatively. The median time to failure for all hips was 8.8 years, the average time to failure was 9.2 years, and $75 \%$ of failures occurred by 13.0 years. The most common failure mechanisms were due to the cup $(5.0 \%)$, dislocation $(3.2 \%)$, cup and stem $(1.7 \%)$, infection $(0.4 \%)$, and the stem $(0.4 \%)$. Based on the most common failure mechanisms, it is recommended to evaluate patients at the 6 months, 1 year, 3 years, 7 years, 10 years, 12 years, 18 years, and 25 years postoperatively.
\end{abstract}

\section{Keywords}

Component, Total Hip Arthorplasty, Postoperative, Optimal Follow-Up, Fail, Risk

\section{Introduction}

The Number of total hip arthroplasty (THA) is expected to rise dramatically and, in fact, is already beginning to do so, as a greater portion of the patient population ages [1] [2]. As THA becomes more prevalent, high-volume arthroplasty centers will encounter a sharp and sustained rise in their yearly workload, both in the arthroplasties performed and in the follow-up required to monitor the patient's condition and gather data. If a surgeon currently 
performing 200 per year, he/she would have to perform follow-up on 800 patients in the fifth subsequent year and 1300 patients in the tenth year, assuming a typical follow-up schedule of 2 months, 6 months, one year, and every two or three years hence. This level of follow-up work is nearly impossible to maintain without either an increase in paramedical assistance or a decrease in arthroplasties performed.

One method of alleviating this impending strain on clinical practice is to limit follow-up to time periods when THAs are experientially most likely to fail. Development of such a schedule requires an analysis of the time patients are most at risk for failure for various modalities, including polyethylene wear and osteolysis, infection, instability, pain, fracture, loosening, collapse, and dislocation. The optimal follow-up interval after total knee arthroplasty was determined previously after the review of knee records [3]. In this study, we examined the failure mechanisms and times to failure of 8331 hips at our center to determine when prostheses failed most frequently; from these observations, we propose follow-up schedules that would lessen the clinical burden for surgeons while allowing them to sufficiently monitor their patients in the most critical time frames post-arthroplasty.

\section{Methods}

Between January 15, 1973 through May 9, 2012, 11,336 THAs were performed at our center. Of these, 9939 (87.7\%) were primary operations. 1131 hips were completely lost to follow-up, leaving 8331 primary total hip arthroplasties (THAs) performed on 6769 patients with postoperative follow-up.

There were 3725 females (55.0\%) in this patient population, the average age was 66.2 years (S.D. 12.0, range 17 - 96 years), and the average preoperative body mass index was $30.1 \mathrm{~kg} / \mathrm{m}^{2}$ (S.D. 5.8 , range $16.5-60.3 \mathrm{~kg} / \mathrm{m}^{2}$ ). Osteoarthritis was the diagnosis in 5.910 patients (87.3\%), osteonecrosis in 417 patients (6.2\%), rheumatoid arthritis in 183 patients (2.7\%), osteoporosis in two patients (0.03\%), and other diagnoses in 257 patients (3.8\%). Patients were followed for an average of 7.9 years (S.D. 4.8, range 0.1 - 37.3 years). There were 1826 staged bilateral (21.9\%), 1298 simultaneous bilateral (15.6\%), and 5207 unilateral (62.5\%) THAs in this population.

\subsection{Raw Rates of Failure}

The authors began their analysis with determining the Kaplan-Meier survivorship results for the entire patient population and for each possible failure mechanism or endpoint (infection, fracture, dislocation, radiolucency, stem failure, cup failure, overall aseptic loosening, polyethylene wear, and death). For each group, the authors also examined the average, median, and interquartile ranges of the time to failure. Results of these analyses appear in Table 1 and Figure 1.

\subsection{Risk of Failure at 1 -Year Follow-Up Intervals}

Based on the data above, the authors endeavored to identify when patients are most at risk for failure by quantifying the conditional probability of failure at each one-year interval (Table 1 and Figure 1). This probability can be derived from the actuarial or life table method, which is similar to Kaplan-Meier survivorship analysis except that the actuarial method's primary units are time intervals, not events (Equation (1) and Equation (2)).

Table 1. Fail times by failure mechanism, n = 8331, Primary hips 1969 through 2011.

\begin{tabular}{ccccccccc}
\hline Failure Mechanism & $\mathrm{n}$ count & mean (yrs) & min. & Q1 (25\%) & median (50\%) & Q3 (75\%) & max. (100\%) & Percent \\
\hline Revision Cup \& Stem & 145 & 11.9 & 0.0 & 7.7 & 11.1 & 15.3 & 27.7 & $1.7 \%$ \\
Dislocation (revised or not) & 270 & 1.9 & & & & & & $3.2 \%$ \\
Infection & 30 & 2.8 & 0.0 & 0.2 & 1.1 & 3.3 & 18.4 & $0.4 \%$ \\
Revision Cup & 412 & 9.4 & 0.0 & 5.1 & 9.2 & 13.2 & 27.9 & $4.9 \%$ \\
Revision Stem & 29 & 5.3 & 0.0 & 2.6 & 4.9 & 6.8 & 11.0 & $0.3 \%$ \\
Revision Poly Wear & 4 & 11.7 & 10.8 & 10.9 & 11.4 & 12.4 & 13.0 & $0.0 \%$ \\
Fracture & 6 & 7.6 & & & & & 14.4 & $0.1 \%$ \\
Death & 2786 & 10.0 & 0.0 & 5.1 & 9.0 & 13.8 & 37.3 & $33.4 \%$ \\
Any revision & 694 & 9.2 & 6.1 & 4.7 & 8.8 & 13.0 & 27.9 & $8.3 \%$ \\
Non-fail & 7637 & 7.0 & 0.0 & 2.1 & 5.5 & 10.2 & 37.3 & $91.7 \%$ \\
\hline
\end{tabular}




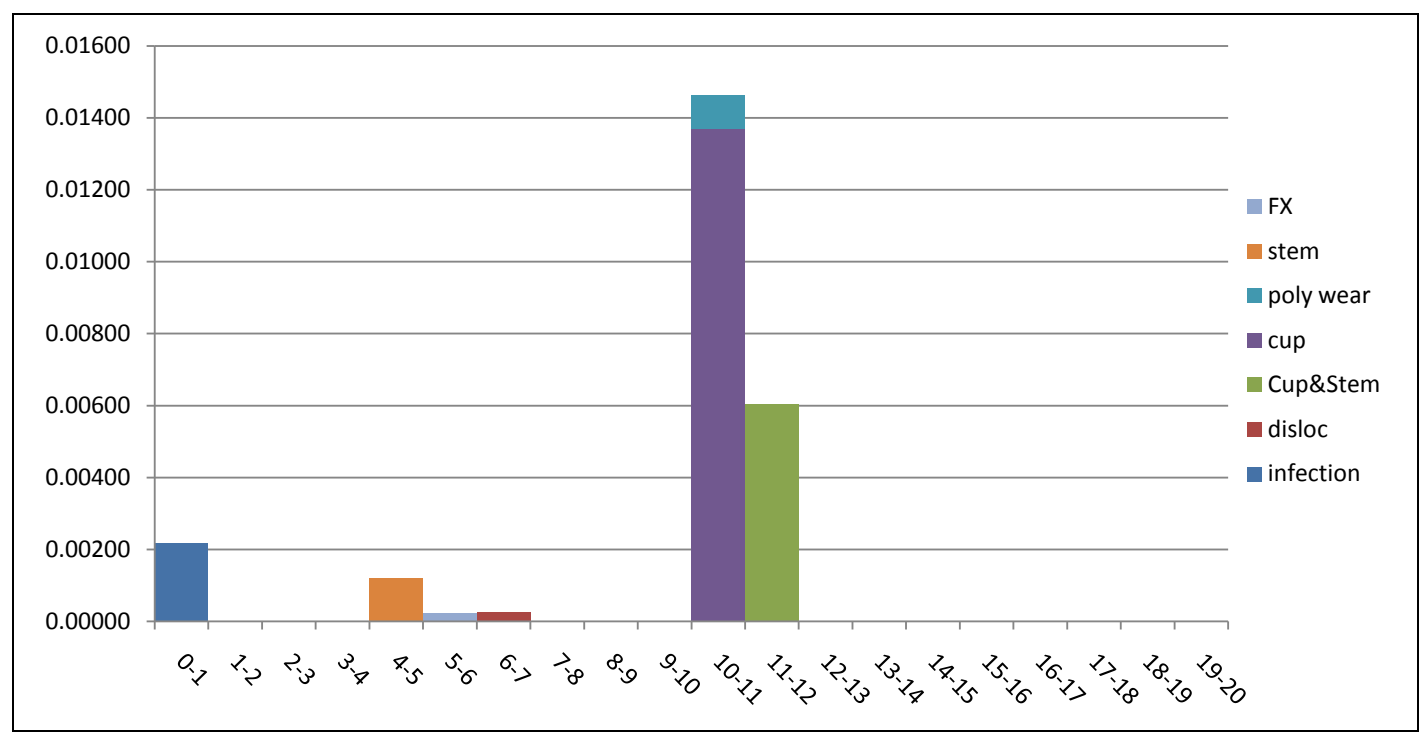

Figure 1. Highest conditional probability of failure for each failure mechanisms at specific time intervals.

$$
\hat{S}\left(t_{i}\right)=\prod_{j=1}^{i}\left(1-\frac{d_{j}}{n_{j}}\right)
$$

Equation (1). Kaplan-Meier procedure. Here:

$\prod=$ product operator (similar to $\Sigma$ in summation, with exception that each iteration is multiplied instead of summed)

$\hat{S}\left(t_{i}\right)=$ survivorship probability at time $t_{i}$

$i=$ time in integer years

$j=$ an event occurring at integer or decimal years

$d_{j}=$ hips failed during event $j$

$n_{j}=$ hips present just prior to event $j$

$$
\hat{S}(t)=\prod_{t_{i}<t} \frac{n_{i}-d_{i}}{n_{i}}
$$

Equation (2). Actuarial or life table procedure. Here:

$\prod=$ product operator

$S(t)=$ survivorship probability at time $t$ (given by the cumulative product of all time intervals that proceed time $t$ )

$t_{i}=$ time interval just prior to current time $t$

$n_{i}=$ hips present at beginning of $t_{i}$

$d_{i}=$ hips failed or in patients who die within $t_{i}$

From the actuarial equation (Equation (2)), the authors could determine $\hat{q}_{i}$, which represents the conditional probability of a predefined endpoint (death or failure) occurring within time interval $t_{i}$ (Equation (3)).

$$
\hat{q}_{i}=\frac{d_{i}}{n_{i}}
$$

Equation (3). Conditional probability of failure. Here:

$\hat{q}_{i}=$ conditional probability of endpoint occurring in $t_{i}$

$d_{i}=$ hips failed or deceased within $t_{i}$

$n_{i}=$ hips present at beginning of $t_{i}$

When the $q_{i}$ values for each endpoint at each time interval are examined, it is possible to determine when most endpoint events will occur, as represented by a peak or peaks (also known as local maxima) of $\hat{q}_{i}$ values. 
From these time periods shown to have peaks of conditional probabilities of failure, the authors devised follow-up schedules that maximized clinical evaluation during such intervals and minimized evaluation outside of those intervals. The derivation of these schedules began with Equation (4):

$$
E_{t}=\sum_{t=0.5}^{t}\left(N_{t}-D_{t}-F_{t}-I_{t}-\cdots\right)
$$

Equation (4). Follow-up load per time period. Here:

$E_{t}=$ expected number of follow-up visits in one time period $t_{i}$

$\sum=$ summation operation

$t=$ time interval just prior to the current time interval $t_{i}$ (at the authors' center, either 6 months or 1, 3, 5, 7, $10,12,15,17,20,22$, or 25 years)

$N_{t}=$ new THAs performed during $t_{i}$ requiring post-op follow-up

$D_{t}=$ hips in patients who die during $t_{i}$

$F_{t}=$ aseptic failures during $t_{i}$

$I_{t}=$ infections during $t_{i}$

$\ldots=$ other failure mechanisms

The peaks in conditional probability can be mathematically derived by taking the first derivative of the best fit curve of these possibilities over time. This best fit curve can provide the closest approximation with a balance of simplicity by using a cubic polynomial equation (a function with a polynomial of degree three). In the authors' analysis, this best-fit curve is expressed in Equation (5):

$$
P=a t^{3}+b t^{2}+c t+d
$$

Equation (5). Cubic polynomial equation of the best-fit curve for conditional probabilities. Here, $a, b$, $c$, and $d$ can be determined by common graphics or spreadsheet software; the authors used matrix algebra similar to that used in linear regression.

The first derivation of Equation (5) results in Equation (6):

$$
\frac{\mathrm{d} P}{\mathrm{~d} t}=3 a t^{2}+2 b t+c
$$

Equation (6). Quadratic polynomial equation, the first derivative of Equation (5).

Setting this equation equal to zero, it is possible to determine the local maxima and minima of the original conditional probability equation (Equation (5)), and thus the time intervals that exhibit the highest and lowest probabilities of failure. The zeros of this equation can be determined through the quadratic formula, Equation (7):

$$
t=\frac{-b \pm \sqrt{b^{2}-4 a c}}{2 a}
$$

Equation (7). Quadratic formula for determining maximum conditional probabilities of reaching an endpoint, whether failure or death. Here, $a, b$, and c are found in Equation (6).

\section{Results}

The most common failure mechanisms in group of 8331 THAs were acetabular cup failure only (5.0\% of total population), dislocation (3.2\%), cup and stem failure (1.7\%), infection ( $0.4 \%)$, and femoral stem failure only $(0.4 \%)$.

As a population, the proportion of hip failures peaked at 10 years and at the subsequent two follow-up periods (Figure 1), the solution to Equation (6), peak failure time, was 11 years (Figure 2). 129 hips (2.1\% of remaining hips) failed at 10 years, 94 hips (1.7\%) failed at 12 years, and 78 hips (1.7\%) failed at 15 years. All other follow-up periods used in this study showed a failure rate of $1.4 \%$ or less of remaining hips (Table 2).

As can be seen in Table 2, most failure mechanisms for THA in our center differed in their schedule profiles; that is, they failed at different follow-up intervals than those of the entire population and those of the other mechanisms. The median time to failure for all hips was 8.8 years, the average time to failure was 9.2 years, and $75 \%$ of failures occurred by 13.0 years. In contrast, femoral stem failures occurred at a median of 4.9 years and a 


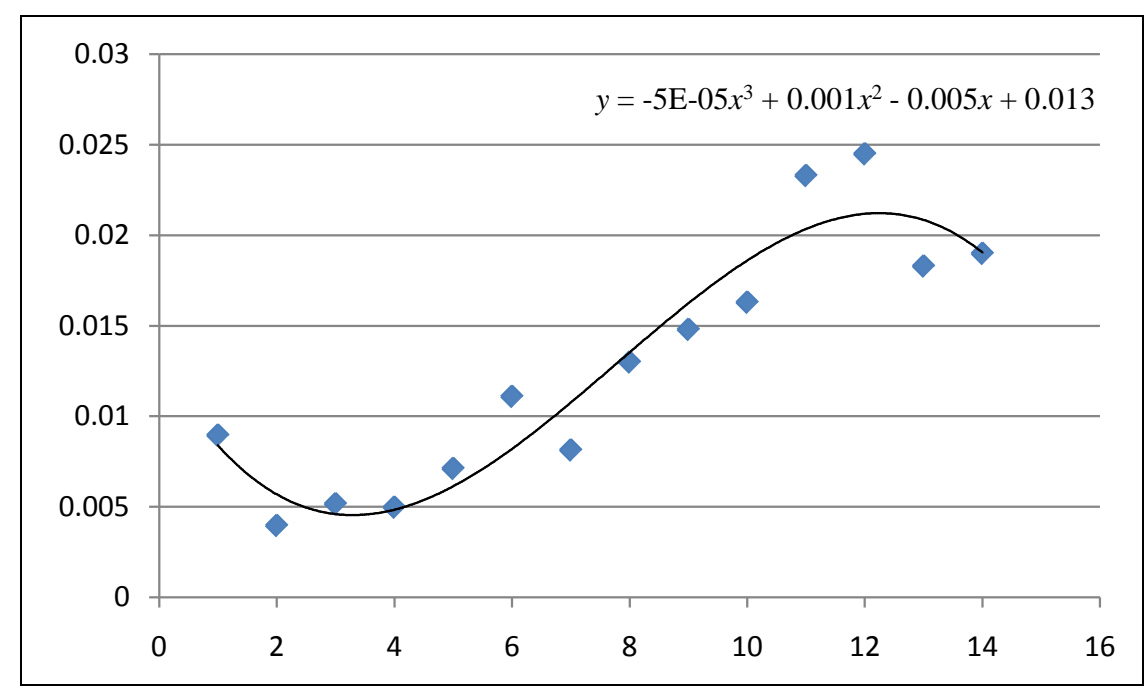

Figure 2. Cubic polynomial equation of the best-fit curve for conditional probabilities of failure (Equation (5)) for all failure mechanisms combined (the solution is 11 years).

Table 2. Probabilities of failure at specific times postoperatively.

\begin{tabular}{|c|c|c|c|c|c|c|}
\hline Time Interval & Failures & $n$ remaining & $\begin{array}{l}\text { Failure rate from } \\
\text { actual count }\end{array}$ & $\begin{array}{l}\text { Conditional probability } \\
\text { of failure }\end{array}$ & $\begin{array}{l}\text { Failure rate out } \\
\text { of max. possible }\end{array}$ & $\begin{array}{l}\text { Maximum number } \\
\text { of THAs remaining }\end{array}$ \\
\hline 6 months & 57 & 8299 & $0.7 \%$ & - & $0.6 \%$ & 9943 \\
\hline 1 year & 9 & 7921 & $0.1 \%$ & $0.9 \%$ & $0.1 \%$ & 9943 \\
\hline 3 years & 58 & 7129 & $0.8 \%$ & $0.5 \%$ & $0.7 \%$ & 8847 \\
\hline 5 years & 64 & 5900 & $1.1 \%$ & $0.7 \%$ & $0.8 \%$ & 8068 \\
\hline 7 years & 82 & 4753 & $1.7 \%$ & $0.8 \%$ & $1.1 \%$ & 7305 \\
\hline 10 years & 129 & 3650 & $3.5 \%$ & $1.6 \%$ & $2.1 \%$ & 6166 \\
\hline 12 years & 94 & 2348 & * & $2.5 \%$ & $1.7 \%$ & 5439 \\
\hline 15 years & 78 & 1652 & $*$ & * & $1.7 \%$ & 4537 \\
\hline 17 years & 42 & 948 & * & * & $1.1 \%$ & 3903 \\
\hline 20 years & 41 & 604 & * & * & $1.4 \%$ & 3016 \\
\hline 22 years & 17 & 299 & * & $*$ & $0.8 \%$ & 2035 \\
\hline 25 years & 17 & 175 & * & $*$ & $0.9 \%$ & 1823 \\
\hline $25^{+}$years & 6 & 94 & * & * & $0.4 \%$ & 1652 \\
\hline
\end{tabular}

* Omitted since failure is overestimated due to follow-up rate, we referred to determine max possible failure rate to peak rate. -: conditional probability of failure was annual for this comparison.

mean of 5.3 years, and $75 \%$ of failures occurred by 6.8 years; acetabular cup failures occurred at a median of 9.2 years and an average of 9.4 years, and $75 \%$ of failures occurred by 13.2 years; polyethylene wear failures occurred at a median of 11.4 years and a mean of 11.7 years, and $75 \%$ of failures occurred at 12.4 years; and infection occurred at a median of 1.1 years and a mean of 2.8 years, and $75 \%$ of failures occurred by 3.3 years.

Based on the most common failure mechanisms, the data suggest that the overall THA patient population should be seen at 6 months, 1 year, 3 years, 7 years, 10 years, 12 years, 18 years, and 25 years after the index surgery. This schedule would cut nearly in half the follow-up schedule used currently by the authors at their center while still allowing for sufficient opportunity to monitor for expected failures.

\section{Discussion}

The number of primary and revision total hip and knee arthroplasties is expected to rise dramatically as more patients wish to remain active [1]. The concomitant increase in the workload of arthroplasty surgeons requires a 
reconsideration of the priorities of large specialty centers with regard to follow-up of a growing population of THA patients. Although these centers' long-term data are essential in tracking the longevity of prostheses and treatment options both old and new, it will become less feasible to record data with such frequency as is found at the authors' center, where patients are followed at 2 months, 6 months, and 1, 3, 5, 7, 10, 12, 15, 17, and 20 years after primary and revision arthroplasty. There is a pertinent need to balance the benefits of frequent and lengthy follow-up with the limited time and resources of surgeons and their staff, which is one of the main concerns of the present study.

The authors found data that support a revised schedule of follow-up for total hip arthroplasty that maximizes the gathering of relevant patient data while minimizing the number of follow-up appointments required of the typical THA patient. The data apply to the patient population as a whole; while further research may support the need for specific schedules for certain patients, the data here do not confer a predictive power on early (6 months to 1 year) increased pain or higher BMI $\left(>41 \mathrm{~kg} / \mathrm{m}^{2}\right)$.

Of concern for clinical research is the loss of potentially valuable follow-up data if the contracted observation schedule proposed above is adopted. Total hip arthroplasty has enjoyed highly satisfactory rates of success since their establishment and throughout their development [hip survivorship papers], but specific issues such as the most advantageous surgical approach remain unresolved due to a lack of solid data favoring one option over the others [4]. Most reputable results on survivorship are reported in retrospective studies originating in large THA speciality centers [5]-[8]. Many of these studies report clinical scores throughout the follow-up period; these results have been invaluable in determining differences between prostheses, cement techniques, approaches and other variables that cannot be captured by survivorship analysis. With the constant stream of new total hip prostheses continuing to reach the market, the orthopedic community would do well to monitor long-term success of these implants through randomized controlled trials and meta-analyses [9].

This trove of clinical information, however, must be balanced with the demands of data gathering on large surgery centers. The proposed schedules are an attempt to balance between monitoring changes and differences in large patient populations and allowing surgeons to continue to meet the surgical demands of their patients without an inhibitively large follow-up caseload. These findings aim to assist the clinician in using follow-up resources at the most relevant points, allowing for a full, yet efficient, postoperative analysis of the patient.

This follow-up schedule is unique to THA and should not be applied to practice in total knee arthroplasty (TKA). A study at the authors' center, concurrent with the present analysis, of optimal follow-up intervals following total knee arthroplasty found predictive value in the 6-month pain subscore and in preoperative BMI reported as previously [3]. Early follow-up intervals in our study do not demonstrate a strong predictive value of 6-month, 1-year, and 3-year clinical evaluations in helping to predict the future success or failure of a THA, nor is it able to propose a variant follow-up schedule for patients with BMI greater than $41 \mathrm{~kg} / \mathrm{m}^{2}$, contrary to the predictive value that may be found in TKA. Early follow-up, however, should by no means be sacrificed to aid in decreased workload. These data points can help the evaluating surgeon anticipate future actions; these periods are also known intuitively to assist in monitoring for deep periprosthetic joint infection [10], and the results of this study (afflicted THAs infected at a median of 1.1 years) give further credence to that belief.

The proposed decrease in frequency of follow-up in clinic after total hip arthroplasty may, if the operating surgeon desires, be supplemented with questionnaires given over the phone. The Oxford, WOMAC, and SF-36 forms have been validated for such use [11]-[16], and while it is more difficult for a patient to measure hip ROM than it is to measure the same in a knee [17], it is possible to estimate hip flexion based on questions in WOMAC or the Harris hip score such as difficulty in putting on socks and tying shoes [18]. The postulated schedules in this study attempt to decrease the workload of the operating surgeon in clinical follow-up; if the surgeon wishes for detailed information on a patient's status in between follow-up visits, a telephone questionnaire could provide sufficient information without the need for a physical examination and the resulting use of clinic time and resources.

This study is, to the authors' knowledge, the first to propose efficient schedules of follow-up based on data from such a large sample of total hip arthroplasties. The findings here are valuable for both small community hospitals and large joint specialty centers, as both will find increasing populations of patients requiring hip replacement and will need to manage finite resources of personnel and time.

There are some limitations in the current study. First, it was not a prospective study and there were wide ranges of prosthesis including different sizes of cups, femoral stems and liners. Second, the committed surgeons were similar and rarely changed but were not exactly the same. This study was performed at one large surgery 
center with a relatively stable patient population, so its proposals may need to be adapted for tertiary care centers whose patients do not reside in the surrounding area and for whom attendance at follow-up is more difficult than it is for our population. This limitation mostly applies to the necessity of early follow-up, however, as the longer intervals between later follow-up periods would allow for greater flexibility for those patients who require follow-up but reside far from the clinic.

These findings, while important to the study of effective follow-up for patient populations, should be verified by clinicians in other practice settings. In addition, studies involving further variables, such as age at time of surgery, extent of arthritic damage, gender, and diagnosis, may determine other schedules that the current study did not elucidate. The results and conclusions made here provide a stable foundation for future work that may provide even more personalized schedules of follow-up so that clinical resources are expended with greater efficiency and greater benefit to the patient.

\section{References}

[1] Kurtz, S., Ong, K., Lau, E., Mowat, F. and Halpern, M. (2007) Projections of Primary and Revision Hip and Knee Arthroplasty in the United States from 2005 to 2030. Journal of Bone and Joint Surgery (American), 89, 780-785. http://dx.doi.org/10.2106/JBJS.F.00222

[2] Kurtz, S., Mowat, F., Ong, K., Chan, N., Lau, E. and Halpern, M. (2005) Prevalence of Primary and Revision Total Hip and Knee Arthroplasty in the United States from 1990 through 2002. Journal of Bone and Joint Surgery (American), 87, 1487-1497. http://dx.doi.org/10.2106/jbjs.d.02441

[3] Meding, J.B., Ritter, M.A., Davis, K.E. and Farris, A. (2013) Meeting Increased Demand for Total Knee Replacement and Follow-Up: Determining Optimal Follow-Up. Bone and Joint Journal, 95, 1484-1489.

http://dx.doi.org/10.1302/0301-620X.95B11.32467

[4] Jolles, B.M. and Bogoch, E.R. (2006) Posterior versus Lateral Surgical Approach for Total Hip Arthroplasty in Adults with Osteoarthritis. Cochrane Database of Systematic Reviews, 19, CD003828.

[5] Berry, D.J., Harmsen, W.S., Cabanela, M.E. and Moorey, B.F. (2002) Twenty-Five-Year Survivorship of Two Thousand Consecutive Primary Charnley Total Hip Replacements. Journal of Bone and Joint Surgery (American), 84, 171177.

[6] Callaghan, J.J., Bracha, P., Liu, S.S., Piyaworakhun, S., Goetz, D.D. and Johnston, R.C. (2009) Survivorship of a Charnley Total Hip Arthroplasty: A Concise Follow-Up, at a Minimum of Thirty-Five Years, of Previous Reports. Journal of Bone and Joint Surgery (American), 91, 2617-2621. http://dx.doi.org/10.2106/JBJS.H.01201

[7] Klapach, A.S., Callaghan, J.J., Goetz, D.D., Olejniczak, J.P. and Johnston, R.C. (2001) Charnley Total Hip Arthroplasty with Use of Improved Cementing Techniques. Journal of Bone and Joint Surgery (American), 83, 1840-1848.

[8] Mallory, T.H., Lombardi Jr., A,V., Leith, J.R., et al. (2001) Minimal 10-Year Results of a Tapered Cementless Femoral Component in Total Hip Arthroplasty. Journal of Arthroplasty, 16, 49-54. http://dx.doi.org/10.1054/arth.2001.28721

[9] Sharma, R., Vannabouathong, C., Bains, S., Marshall, A., MacDonald, S.J., Parvizi, J. and Bhandari, M. (2011) MetaAnalyses in Joint Arthroplasty: A Review of Quantity, Quality, and Impact. Journal of Bone and Joint Surgery (American), 93, 2304-2309. http://dx.doi.org/10.2106/jbjs.j.01289

[10] Tsukayama, D.T., Estrada, R. and Gustilo, R.B. (1996) Infection after Total Hip Arthroplasty. A Study of the Treatment of One Hundred and Six Infection. Journal of Bone and Joint Surgery (American), 78, 512-523.

[11] Angst, F., Ewert, T., Lehmann, S., Aeschlimann, A. and Stucki, G. (2005) The Factor Subdimensions of the Western Ontario and McMaster Universities Osteoarthritis Index (WOMAC) Help to Specify Hip and Knee Osteoarthritis. A Prospective Evaluation and Validation Study. Journal of Rheumatology, 32, 1324-1330.

[12] Bellamy, N., Buchanan, W.W., Goldsmith, C.H., Campbell, J. and Stitt, L.W. (1998) Validation Study of WOMAC: A Health Status Instrument for Measuring Clinically-Important Patient-Relevant Outcomes Following Total Hip or Knee Arthroplasty in Osteoarthritis. Journal of Orthopedics \& Rheumatology, 1, 95-108.

[13] Dawson, J., Fitzpatrick, R., Carr, A. and Murray, D. (1996) Questionnaire on the Perceptions of Patients about Total Hip Replacement. Journal of Bone and Joint Surgery (British), 78, 185-190.

[14] Dawson, J., Fitzpatrick, R., Frost, S., Gundle, R., McLardy-Smith, P. and Murray, D. (2001) Evidence for the Validity of a Patient-Based Instrument for Assessment of Outcome after Revision Hip Replacement. Journal of Bone and Joint Surgery (British), 83, 1125-1129. http://dx.doi.org/10.1302/0301-620X.83B8.11643

[15] Ware Jr., J.E., Keller, S.D., Hatoum, T.H. and Kong, S.X. (1999) The SF-36 Arthritis-Specific Health Index (ASHI): I. Development and Cross-Validation of Scoring Algorithms. Medical Care, 37, MS40-MS50.

http://dx.doi.org/10.1097/00005650-199905001-00004 
[16] Ware Jr., J.E., Kosinski, M. and Keller, S.D. (1994) SF-36 Physical and Mental Health Summary Scales: A Users Manual. The Health Institute, New England Medical Center, Boston.

[17] Khanna, G., Singh, J.A., Pomeroy, D.L. and Gioe, T.J. (2011) Comparison of Patient-Reported and Clinician-Assessed Outcomes Following Total Knee Arthroplasty. Journal of Bone and Joint Surgery (American), 93, e117(1)-(7).

[18] McGrory, B.J., Freiberg, A.A., Shinar, A.A. and Harris, W.H. (1996) Correlation of Measured Range of Hip Motion Following Total Hip Arthroplasty and Responses to a Questionnaire. Journal of Arthroplasty, 11, 565-571. http://dx.doi.org/10.1016/S0883-5403(96)80111-2 